\title{
Hydroxyapatite delivery to dentine tubules using carboxymethyl cellulose dental hydrogel for treatment of dentine hypersensitivity
}

\author{
Alexander Sadiasa ${ }^{1}$, Rose Ann Franco ${ }^{1}$, Hyung Seok Seo ${ }^{2}$, Byong Taek Lee ${ }^{{ }^{*}}$ \\ ${ }^{1}$ Department of Biomedical Science, Soonchunhyang University, Ssangyong-dong, Cheonan, South Korea \\ ${ }^{2}$ Department of Exercise Prescription, Konyang University, Dahak Ro Nae-dong, Non San City, South Korea \\ Email: ${ }^{*}$ lbt@sch.ac.kr
}

Received 28 August 2013; revised 25 September 2013; accepted 14 October 2013

Copyright (C) 2013 Alexander Sadiasa et al. This is an open access article distributed under the Creative Commons Attribution License, which permits unrestricted use, distribution, and reproduction in any medium, provided the original work is properly cited.

\begin{abstract}
This study evaluated the effectiveness of carboxymethyl cellulose (CMC) hydrogel as a dental gel in delivering hydroxyapatite (HAp) to dentine tubules and reducing/eliminating dental hypersensitivity. The hydrogel was prepared by mixing solutions of $\mathrm{CMC} /$ glycerol and distilled water/sorbitol then modified to contain $0 \%, 10 \%, 20 \%$ and $30 \%$ HAp. The $\mathrm{pH}$ of the hydrogels decreased and viscosity increased with increasing HAp content. A viability assay was performed to determine the cytotoxicity of the hydrogel samples and proliferation/adhesion behavior of cells cultured on the hydrogel surface. The samples promoted cell proliferation and became more biocompatible with the addition of HAp. Dentin discs were prepared and then treated with the fabricated hydrogels. Occlusion of the dentine tubules was observed by scanning electron microscopy before and after treatment. Blocking of the dentin tubules was markedly affected by the addition of HAp to the hydrogel samples that can result in possible reduction or elimination of hypersensitivity.
\end{abstract}

Keywords: CMC; Hydroxyapatite; Teeth; Dental; Hypersensitivity

\section{INTRODUCTION}

One of the common clinical problems concerning human teeth is the occurrence of dentine hypersensitivity [1-3]. It is considered to be an oral health concern among adults worldwide [4]. The incidence of hypersensitivity ranges from $10 \%-30 \%$ of the general population [5]. Dentine hypersensitivity is defined as a transient pain arising in

"Corresponding author. response to chemical, thermal, tactile or osmotic stimuli [6]. This problem can be caused by the exposure of dentine tubules as a result of enamel wear or gingival recession, which allows intradentinal fluid movement and leads to dentinal hypersensitivity [7-9]. This causes an excitation in the nerves inside the tooth exposed through the openings of the dentine tubules [10]. Although dentinal hypersensitivity is not considered life-threatening or a serious dental injury, the resulting uncomfortable and unpleasant sensation, variously described as dull or sharp, vague or specific and intermittent or constant, may dictate the diet adopted by those affected, limiting their choices of food [5].

Procedures aimed at reducing or eliminating dental hypersensitivity range from simple home desensitizing therapies to more complicated clinical processes such as surgery, pulpectomy or laser treatments [11,12]. An ideal desensitizing agent should not irritate or endanger the integrity of the pulp, should be painless immediately upon application and thereafter, be easy to apply, display rapid effect, be long-lasting or permanent and should not damage the teeth or gums, such as by discoloration. Many treatments have been devised according to these criteria, but no gold standard has been established [13].

One of the materials being utilized to treat hypersensitivity is hydroxyapatite (HAp). HAp is considered to be chemically similar to enamel. The ultra-structure of dental enamel consists of inorganic components distributed as rod and prisms of hexagonal HAp crystals [14]. HAp is also used as a component of bone substitutes and has become a mainstay material for clinical use due to its bioactivity and biocompatibility [15]. The similarities between HAp and human enamel have spurred interest in use of HAp in the remediation of damaged enamel [1618]. The use of HAp as a filler in dental restoration presents advantages that include intrinsic radio-opaque re- 
sponse, enhanced polishing ability, improved wear performance and reduced expense, compared to materials commonly used in dental health care [19].

The aim of the present study was the delivery of HAp to dentine tubules using carboxymethyl cellulose (CMC) hydrogel. CMC is a natural biodegradable and biocompatible anionic polymer obtained from natural cellulose by chemical modification. CMC polymers are often used as thickeners and additives in the food and chemical industries [20,21]. CMC is enriched in hydroxyl groups that can be used to prepare hydrogels easily with fascinating and adjustable structures and properties to suit the required applications [22]. In one study, CMC hydrogel was used to deliver stannous fluoride to control dentinal hypersensitivity [23]. Presently, HAp-containing CMC hydrogels were characterized physically and chemically, and were tested for in vitro biocompatibility. As well, the HAp-amended hydrogels were applied to dentine samples as a means of occluding dentinal tubules.

\section{EXPERIMENTAL PROCEDURES}

\subsection{Hydrogel Preparation}

The ingredients utilized in the study are summarized in Table 1. Hydrogels were prepared using CMC mixed with glycerol in a beaker using a magnetic stirrer until homogeneous. As the mixing of glycerol/CMC was being conducted, a solution of distilled water and sorbitol was also stirred until homogeneous. The two solutions were combined and continuously mixed for $1 \mathrm{~h}$.

CMC hydrogels were also prepared containing varying ratios of Hap as shown in Table 2. HAp powder was synthesized using microwave-hydrothermal method. Calcium nitrate tetrahydrate $\left(\mathrm{Ca}\left(\mathrm{NO}_{3}\right)_{2} \cdot 4 \mathrm{H}_{2} \mathrm{O}, 98.5 \%\right.$; SAMCHUN Chemicals, Seoul, South Korea) and ammonium phosphate $\left(\left(\mathrm{NH}_{4}\right)_{2} \mathrm{HPO}_{4}, 98.5 \%\right.$; SAMCHUN Chemicals) were used as the starting materials in this preparation. The materials were mixed with water separately and stirred until completely dissolved. The solutions were prepared with the reactants at a weight molar ratio of $1.17-1.77$. The solutions were combined and stirred for $10 \mathrm{~min}$. The resulting single solution was transferred to a closed-vessel microwave device constructed of perfluoralkoxy (PFA) Teflon and exposed to $1200 \mathrm{~W}$ of output power generated by a dual magnetron device using $2.45 \mathrm{GHz}$ frequency microwave radiation. Three milliliters of deionized water was added to the resulting mixture, followed by a three-step procedure. The first step was a warming-up process generated at $250 \mathrm{~W}$ for $2 \mathrm{~min}$. The second step was a main reaction process generated at $250-650$

Table 1. Composition of CMC Hydrogel.

\begin{tabular}{ccccc}
\hline & CMC & Glycerol & Sorbitol & $\mathbf{d H}_{2} \mathbf{O}$ \\
\hline CMC Hydrogel & $2 \%$ & $15 \%$ & $25 \%$ & $58 \%$ \\
\hline
\end{tabular}

Table 2. Composition of the HAp-modified CMC hydrogel.

\begin{tabular}{ccccc}
\hline & CHAp0 & CHAp10 & CHAp20 & CHAp30 \\
\hline $\begin{array}{c}\text { CMC + Glycerol + } \\
\text { Sorbitol (w/v) }\end{array}$ & $42 \%$ & $32 \%$ & $22 \%$ & $12 \%$ \\
Hap (w/v) & $0 \%$ & $10 \%$ & $20 \%$ & $30 \%$ \\
$\mathbf{d H}_{2} \mathbf{O}$ (w/v) & $58 \%$ & $58 \%$ & $58 \%$ & $58 \%$ \\
\hline
\end{tabular}

$\mathrm{W}$ for $4 \mathrm{~min}$. The final step was a 20 -min cooling process. After completion of HAp synthesis, both the elimination of residuals in products and the crystallization of powders were achieved by microwave heating at $250 \mathrm{~W}$ for $3 \mathrm{~min}$. Samples containing $0 \%, 10 \%, 20 \%$ and $30 \%$ HAp were prepared and used in this experiment.

\subsection{Physical and Chemical Analyses}

The fabricated hydrogels were analyzed by attenuated reflectance Fourier transform infrared spectroscopy (FTIR) using a Spectrum GX apparatus (PerkinElmer, New Jersey, USA). The IR spectra of the samples were measured over a wavelength range of $4000-500 \mathrm{~cm}^{-1}$, and the spectra were collected from 64 scans with a resolution of $4 \mathrm{~cm}^{-1}$. The viscosity of the samples was measured using a Viscotester VT-04F viscometer (Rion, Tokyo, Japan). Three hundred milliliters of each sample was prepared and measured for a certain time range. The $\mathrm{pH}$ of the samples was measured using a PHS-3BW $\mathrm{pH}$ meter (Bante, Shanghai, China). Measurements were conducted five times for each sample. Dilutions $(10 \%)$ of each sample were prepared using phosphate buffered saline (PBS) before measurement.

\subsection{Cell Culture}

M3CT3-E1 cells obtained from Korean Cell Line Bank were maintained following prescribed protocols. Upon confluence, cells were washed with PBS to remove metabolic wastes, media and non-adherent cells prior to detachment with Trypsin-EDTA for 5 minutes. Detached cells were then collected and centrifuged at $1500 \mathrm{rpm}$ for $150 \mathrm{~s}$ and re-suspended with fresh minimum essential medium (MEM) supplemented with $10 \%$ fetal bovine serum and $1 \%$ penicillin/streptomycin antibiotics. Incubation conditions were $37^{\circ} \mathrm{C}$ and $5 \% \mathrm{CO}_{2}$. Medium was replaced every 2 days.

\subsection{Cytotoxicity and Cell Growth Behavior}

Determination of the cytotoxicity of the samples was done according to previously published protocols done in our laboratory with modifications in consideration of the consistency of the samples [24]. Samples were immersed in MEM in 1:3 ratio of sample weight to media volume and agitated at $50 \mathrm{rpm}$ under humidified conditions at $37^{\circ} \mathrm{C}$ for $24 \mathrm{~h}$. Consequently, cells were seeded in wells 
of a 24-well plate, $10^{3}$ cells $/ \mathrm{ml}$, and incubated under the aforementioned conditions for $24 \mathrm{~h}$. After incubation, cells were treated with medium supplemented with extract solution in a 50:50 ratio for 1, 2 and 3 days.

Quantization of cell viability was done by a 3-(4,5dimethylthiazol-2-yl)-2,5-diphenyltetrazolium bromide (MTT)-based assay. MTT substrate $(5 \mathrm{mg} / \mathrm{ml})$ were supplied to cells and incubated for $4 \mathrm{~h}$. The converted formazan salts were dissolved with dimethysulfoxide and the absorbance was read at $595 \mathrm{~nm}$ using a microplate reader (Tecan, Mannedorf, Switzerland). The obtained optical densities were equated with the relative amount of viable cells that were able to convert the substrate.

Live/Dead ${ }^{\circledR}$ cell staining was done during the third day of incubation to visualize the cell growth under prolonged treatment with extract solutions of samples. Stained cells were viewed using a Fluoview 100 confocal laser scanning microscope (Olympus) using $473 \mathrm{~nm}$ and $559 \mathrm{~nm}$ fluorophore filters for Calcein AM and Ethidium homodimer-1, respectively. Viable cells were stained with Calcein AM and fluoresced green, while dead cells were stained with Ethidium homodimer-1 and fluoresced red.

\subsection{Preparation of Dentine Samples}

Caries-free and cleaned human molar teeth were obtained from healthy adults. The teeth samples were submerged in ethanol before being using to obtain dentine disks. The dentine discs were prepared with a thickness of $1.0 \mathrm{~mm}$ by sectioning with a RB 205 MetSaw Loaw High Speed Diamond Cutter (R \& B, Daejeon, South Korea). Occlusal enamel was removed to expose the middle dentine. The sectioned discs were polished then etched with $6 \%$ citric acid for 2 minutes to completely open the dentine tubules. After etching, the samples were ultrasonicated to further expose the dentine occlusions and remove the residual smear layers.

\subsection{Experimental Treatment}

The dentine discs samples were randomly grouped into four groups with each group to be treated with each fabricated samples respectively. The samples were applied as dental gel in the same manner toothpaste is applied to teeth. Dentine specimens were brushed manually using an electric toothbrush operating at 20,000 rpm. Hydrogel $(0.5 \mathrm{~g})$ were applied every treatment and treatments were done three times a day ( $8 \mathrm{am}, 2 \mathrm{pm}$ and $8 \mathrm{pm})$. Every treatments lasts for 2 minutes at room temperature. After every treatment, the dentine discs were washed with deionized water to remove excess samples.

\subsection{Scanning Electron Microscopy (SEM) Observation}

The dentine tubules were observed using a JSM-635 scanning electron microscope (JEOL, Tokyo, Japan). The dentine discs samples were fixed in a sample holder and coated with platinum using a SPI-module Sputter coater operating at $7 \mathrm{~mA}$. The observation was conducted before and after treatment with the hydrogel samples.

\subsection{Statistical Analyses}

The values reported in this study were expressed as the average of three replicates or repetitions unless stated otherwise. The resulting values were analyzed statisticcally using single factor and two-way ANOVA with posthoc correction (Tukey's and Bonferroni method) using GraphPad Prism 5 with a confidence level of $\mathrm{p} \leq 0.05$ to determine statistical significance of each result.

\section{RESULTS}

\subsection{Characteristics of the CMC Hydrogels}

The different components of the hydrogel were mixed and formed a paste-like consistency. Figure 1(a) shows the experimental procedure done to prepare the hydrogel and the resulting hydrogels with and without HAp are depicted in Figures 1(b) and (c), respectively. Complete incorporation of different concentrations of HAp within the hydrogel mixture was shown in the FTIR spectra of the different samples (Figure 2).

All FTIR representative spectra were recorded in the region of $500-4000 \mathrm{~cm}^{-1}$. A broad band ranging from 3000 to 3600 was observed (Figure 2) and could be attributed to the stretch vibration of $\mathrm{O}-\mathrm{H}$ group. The band at 2940 to 2960 was due to carbon-hydrogen bonding (a)

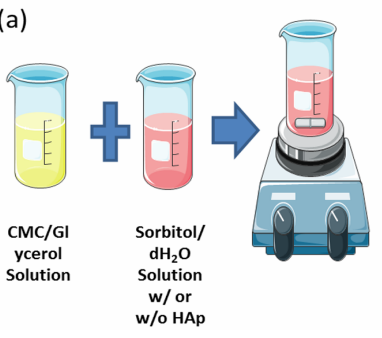

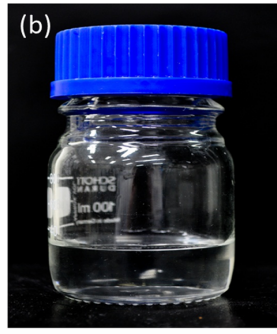

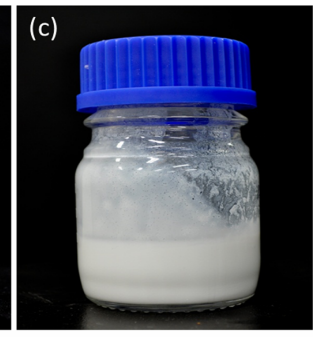

Figure 1. Experimental procedure in the preparation of the hydrogel (a) and photograph of CMC hydrogel without HAp (b) and CMC hydrogel with Hap (c). 


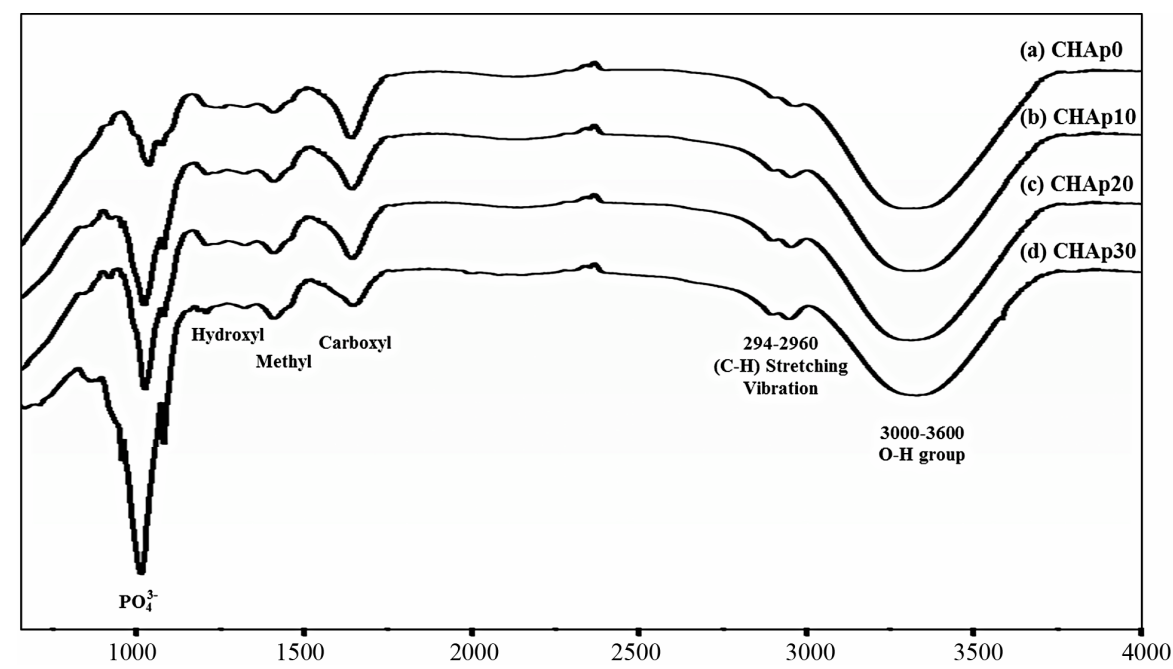

Figure 2. FT-IR spectra of the fabricated CMC hydrogels modified with varying concentrations of HAp.

(C-H) stretching vibration. The carboxyl, methyl and hydroxyl functional groups displayed a wavelength of 1640,1410 and 1320 respectively. The band at 1460 is assigned to the $\mathrm{CO}_{3}^{2-}$. An intense peak was evident at around 1030, consistent with $\mathrm{PO}_{4}^{3-}$. More $\mathrm{PO}_{4}^{3-}$ peaking were seen in the region of 1080, 960 and 870 . The FTIR spectra confirmed the complete blending of the $\mathrm{CMC}$ hydrogels and offered a strong indication that HAp was successfully incorporated in the material $[25,26]$.

The samples had the physical characteristics of toothpaste and could be injected out of a syringe or a tube. With the addition of HAp, the viscosity of the hydrogel changed and varied markedly. The addition of HAp increased the viscosity (Figure 3). After 60 min of testing, final viscosity measurements of $33 \mathrm{dPa} \cdot \mathrm{s}$ for $\mathrm{CHAp} 0$, $105 \mathrm{dPa} \cdot \mathrm{s}$ for CHAp10, 345dPa.s for CHAp20 and 470 CHAp30 were recorded, showing an increasing rate of viscosity as the HAp concentration increased.

Figure 4 shows the $\mathrm{pH}$ measurement of the hydrogels formulated with the various HAp concentrations. Compared to the PBS control, the addition of HAp progresssively decreased the $\mathrm{pH}$ of the hydrogel. Results of the $\mathrm{pH}$ measurement were $7.19 \pm 0.018$ for CHAp0, $6.83 \pm$ 0.019 for CHAp10, $6.63 \pm 0.007$ for CHAp20 and $6.43 \pm$ 0.008 for Chap30 compared to the measured value of 7.25 for PBS.

\subsection{Cell Viability and Proliferation}

The viability of the MC3T3-E1 in connection to the samples was observed using longer incubation time. Cells proliferated better as the HAp concentration was increased in hydrogels after 1, 3 and 5 days of incubation (Figure 5). Significant changes were observed to the samples containing HAp in comparison to that of CHAp0 $(\mathrm{p}<0.005)$. Visual inspection was consistent with unimpeded viabil-

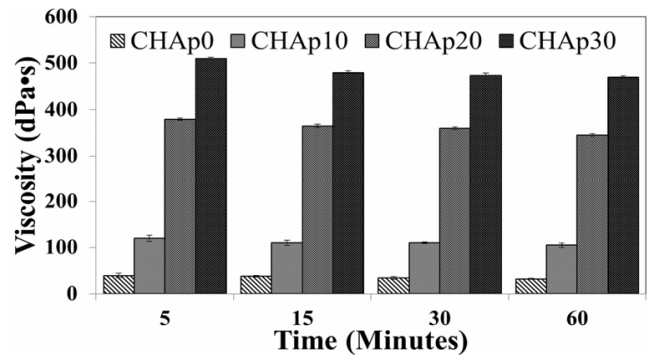

Figure 3. Viscosity measurement of the hydrogel depending on HAp concentration $(\mathrm{p}<0.05 ; \mathrm{n}=3)$.

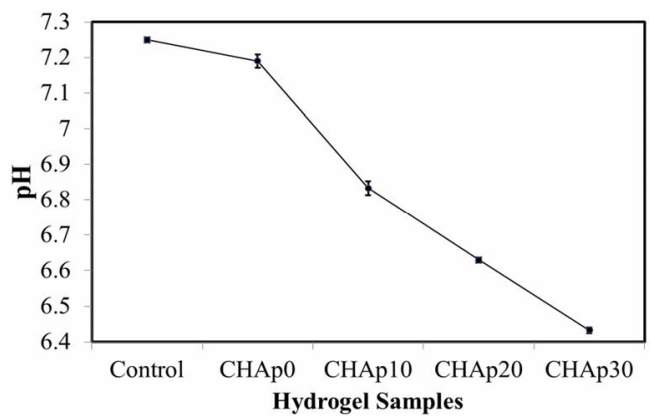

Figure 4. Analysis of the $\mathrm{pH}$ value of the hydrogel depending on Hap concentration $(\mathrm{p}<0.05 ; \mathrm{n}=5)$.

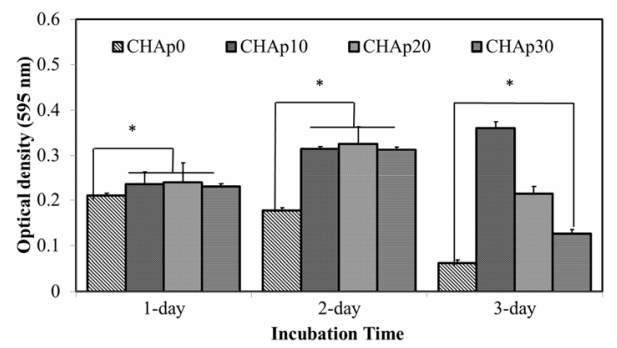

Figure 5. Cell proliferation profiles of M3CT3E1 pre-osteoblast cells on media supplemented with extract solutions of samples. 
ity (Figure 6).

\subsection{SEM/EDS Examination}

Dentine discs were washed with distilled water and subjected to ultrasonication after cutting. The samples were then dried at room temperature and placed in a freeze dryer for further drying prior to SEM observation. SEM revealed exposed open tubules. The dentine surfaces were free of smear layers and almost all of the tubules were completely open (Figure 7).

Two sets of dentine discs were treated with CHAp0 and CHAp30 for 7 days. The dentine surfaces were again observed using SEM after treatment. Hydrogels, whether containing HAp or not, displayed modifications in the surface of the dentine.

The dentine discs treated with CHAp0 (Figures 8(a) and (b)) contained scratches and some debris, possibly due to friction from brushing. The dentine tubules were still left exposed and open, although minute changes were observed due to the presence of debris or roughness, which may be attributed to brushing or dentine remineralization. In the case of CHAp30 (Figures 8(d) and (e)), SEM shows a rougher dentine surface after the treatment. Irregular layers of crystal-like deposits were observed on the surface, which in most cases occluded the exposed dentine tubules. Higher magnification of the tubules revealed completely blocked tubules with crystals attached to walls and openings.

A part of the dentine surface treated with CHAp0 and CHAp30 (Figures 8(c) and (f), respectively) was selected in which energy dispersive X-ray spectroscopy (EDS) analysis was conducted. The presence of elements such as $\mathrm{C}, \mathrm{Ca}, \mathrm{O}, \mathrm{Na}, \mathrm{Si}, \mathrm{P}$, and $\mathrm{S}$ were revealed. Higher and more pronounced peaks indicated the intensity of $\mathrm{C}$, $\mathrm{Ca}, \mathrm{O}$ and $\mathrm{P}$ on the surface of the dentine. An increase in the $\mathrm{CA} / \mathrm{P}$ molar ratio in the dentine surface upon treatment of CHAp30 (2.92) compared to that of CHAp0 (1.59) was evident, indicating that the presence of HAp in the treatment shifted the molar ratio of $\mathrm{Ca} / \mathrm{P}$.

\section{DISCUSSION}

The hypothesis behind the treatments offers two methods in desensitizing dentine: 1) Reduce stimuli-evoked fluid shifts in the dentinal tubules by reducing dentine permeability; and 2) Reduce the ability of the intradental nerves to respond to fluid shifts $[8,27]$. One or both mechanisms must be employed to relieve dentine hypersensitivity. $\mathrm{CMC}$ has been used as base material for artificial saliva
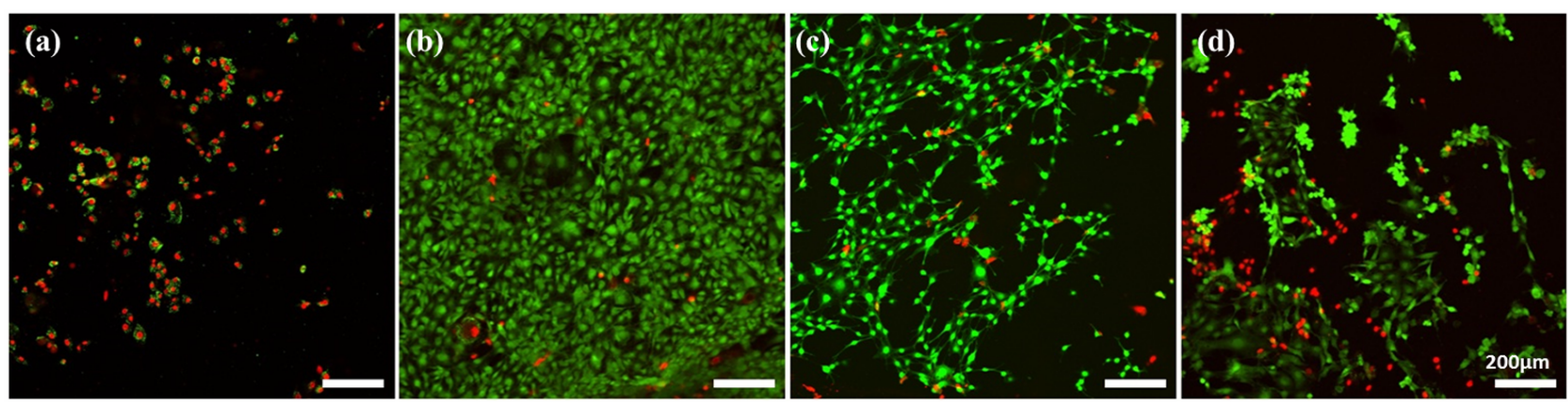

Figure 6. Live/Dead ${ }^{\circledR}$ cell staining of M3CT3-E1 preosteoblast cells grown on polysterene films cultured with media and extract solutions of samples (50:50) after 3 days of culture. CHAp0 (a), CHAp10 (b), CHAp20 (c) and CHAp30 (d).
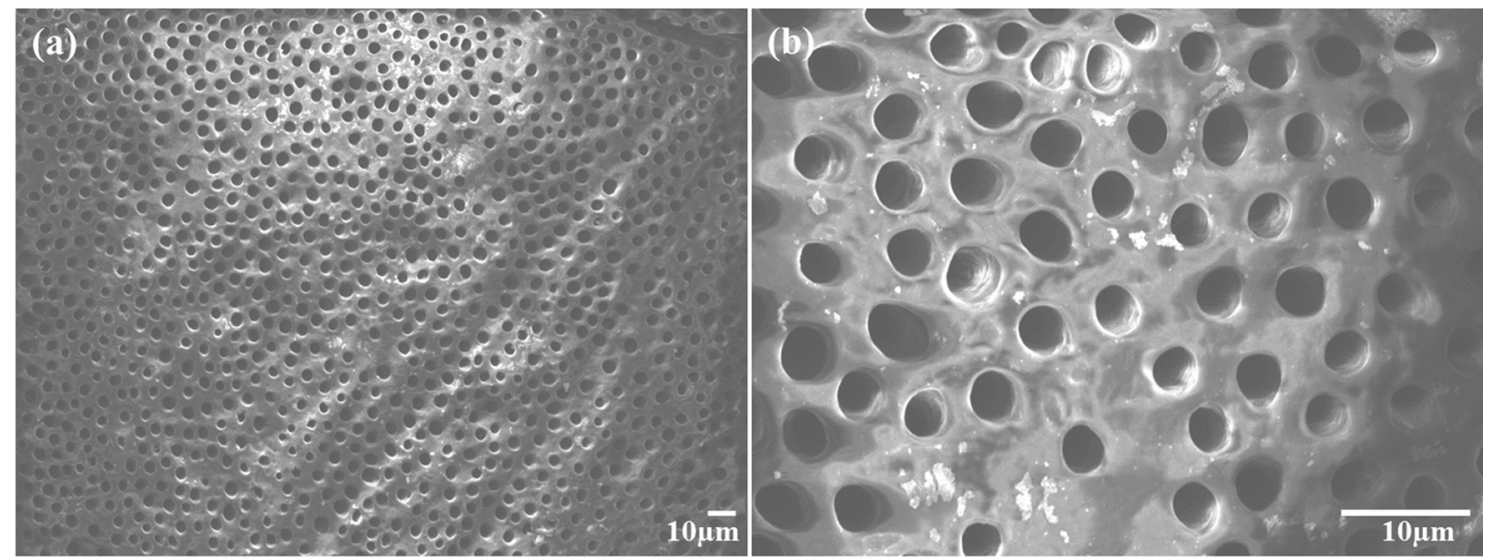

Figure 7. SEM micrograph of the exposed dentine tubules before treatment with the fabricated samples (a) and its higher magnification (b). 

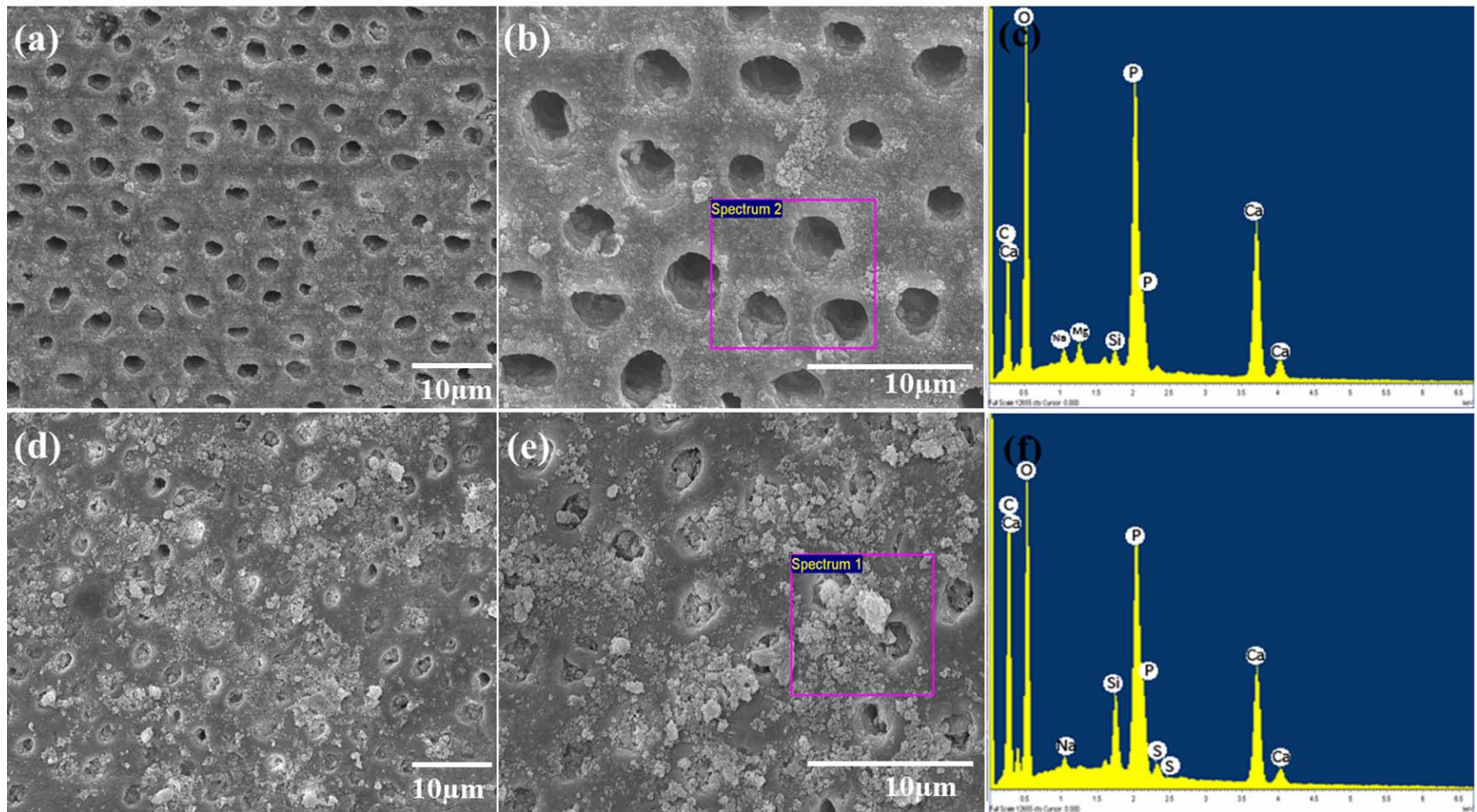

Figure 8. SEM micrograph of the exposed dentine tubules after treatment with CHAp0 (a) with its higher magnification (b) and EDS profile (c) and after treatment with CHAp30 (d) with its higher magnification (e) and EDS profile (f).

and it was reported by Meyer-Lueckel et al. in their study that the remineralising properties of high viscous CMC-based saliva substitutes was improved by the increasing calcium and phosphate concentrations in the fabricated artificial saliva. This was due to the supersaturation of the solution with respect to octacalciumphosphate [28].

In this study, HAp was loaded in CMC hydrogels to deliver it to the surfaces of teeth and to occlude dentine tubules. The FTIR spectra indicated complete blending of the CMC hydrogels and offered convincing evidence that HAp was successfully incorporated in the material, as the fabricated hydrogels presented peaks normally found separately in CMC and HAp.

The ingredients of the hydrogel were adjusted to have the viscosity and feel of toothpaste. CMC, numerous hydroxyl and carboxylic groups and sorbitol and glycerol, which are all known humectants, all have the ability to retain water and so affect viscosity $[29,30]$. Presently, the concentrations of these materials were fixed and that it was observed that the increase in HAp concentration progressively increased caused the viscosity of the fabricated hydrogels. This may be due to the solution being further diluted with the addition of the HAp to the solution or because the dry powders of HAp enhance the water absorption capability of the hydrogel. Also, many properties of HAp are reported to cause increase in viscosity of a slurry [31]. With increase in viscosity, it was observed that the hydrogel seems to be stickier, which may be an advantage as the hydrogel will have a better and longer chance to adhere teeth during application, increasing the probability of HAp being delivered into the dentine tubules.

The addition of HAp to the hydrogel resulted in a progressive decrease in $\mathrm{pH}$. According to Hilgenberg et al., an alkaline $\mathrm{pH}$ has less tendency to cause change to the dental surface and toothpaste with lower $\mathrm{pH}$ can produce a greater change in teeth [32]. In one study, it was demonstrated that when HAp comes in contact with aqueous solution, important amounts of $\mathrm{H}^{+}, \mathrm{Ca}^{+}$and phosphate ions were being released [33]. This may explain the change in $\mathrm{pH}$ in relation to the presence of Hap in the samples.

In vitro biocompatibility testing was conducted, as there is a chance that the treatment will penetrate the dentine tubules and reach the dental pulp before it is totally occluded. Also, the property of the hydrogel may be altered to prepare an appropriate scaffold for pulp regeneration purposes. Health of dental pulp is important as it may cause added pain and further aggravates the case of hypersensitivity [34]. This test can be compared to that of Bakry et al. [9]. Also, it was said that bone and periodontal tissue regeneration depends upon series of biological events starting from cell proliferation to matrix mineralization. This means that the ability to improve cell proliferation is an important factor in developing a biomaterial [35]. Although it was ideal to use human dental pulp stem cell or periodontal ligament cells in this 
experiment, it was thought of to simplify the experimenttal design by using a homogeneous cell line to get a better idea on the effect of the samples to cell behavior as also done in a study conducted by Imazato et al. [36]. In this study, we used pre-osteoblasts MC3T3-E1 cells which is associated with hard tissue formation and frequently used in the evaluation of biomaterials. The fabricated samples were non-cytotoxic upon testing in vitro. After 1 day of incubation, cell growth was significantly higher in wells treated with samples containing HAp (10\%, 20\% and $30 \%$ ) compared to the wells without HAp, indicating that HAp inclusion may have affected initial cell growth or the dissolution of CMC may have induced a cytotoxic effect. Cell viability was observed to increase during the second day for all samples except wells treated with HAp-free sample, where viability was reduced. Among the HAp-loaded samples, there was no significant difference among the optical densities plotted during the second day. Cell growth was declined dramatically during the third day of incubation, except for wells treated with samples containing $10 \% \mathrm{HAp}$, where a slight increase was observed from day 2 to day 3. Confocal laser scanning microscopy images showed congruent findings, as the density of viable cells were relatively higher when treated with extract solution of sample containing $10 \%$ HAp compared to wells with higher concentration of HAp. Consequently, dead cells were relatively denser with higher concentrations of HAp and in wells treated with samples without HAp. These findings suggested that HAp inclusion influence improvement in cell viability but produced reverse effects at higher concentrations. It should be noted that extract solutions were not filtered prior to use since HAp is not soluble in media. The presence of HAp in extract solutions must be evident to provide a more definite conclusion on how it interacts with cells in vitro. The effect of including a ceramic component like HAp generally improved cell growth and interaction with scaffold. However, in a static culture, competition for surface for growth and expansion may have been a factor for apparent findings.

The use of the experimental CMC/HAp hydrogel in occluding dentine tubules were observed to produce a significant reduction of open tubules compared to the CMC hydrogel alone. After 7 days of treatment, the surface of the dentine discs seemed to have formed a smear layer that had a closing effect on the dentinal tubules. The surface contained rough, crystal-like debris scattered all over the dentine and some tubules were completely blocked. The addition of HAp to the hydrogel will supply more calcium and phosphate ions during the remineralization process, thus making the occlusion of dentine tubule possible. During remineralization, the mineraldeprived layers of enamel serves as nucleation sites for new mineral deposition, wherein $\mathrm{Ca}^{2+}$ and $\mathrm{PO}_{4}^{3-}$ ions in saliva settles in as new apatite layers [37]. This scenario was presently supported by the increased in the ratio of calcium and phosphate ions as shown by the EDS profile after the treatment.

The results support the conclusion that CMC/HAp hydrogels can protect dental pulp from open tubules and may help decrease or eliminate dental hypersensitivity. Any substance that causes a decrease in the conductance of dentinal fluid by occluding the dentinal tubules will be able to reduce clinical symptoms of dentine hypersensitivity [8]. Although the results of this study may provide another option for the treatment of dental hypersensitivity, further quantitative studies should be conducted as to the characteristics of the dentine tubules' occlusions and quality. Also, the effect of the hydrogel may vary for every individual response to the treatments may vary from one individual to another and the effectivity of the treatment is very subjective as it may depend on a person's pain threshold [38,39].

\section{CONCLUSION}

The delivery of HAp to dentine surfaces using CMC hydrogels offers a new approach in dealing with dental hypersensitivity. In this study, the hydrogels containing different concentrations of HAp were fabricated and characterized physically and chemically. FTIR spectra showed that HAp was successfully incorporated in the hydrogels and that the presence of HAp increased the viscosity and decreased the $\mathrm{pH}$ of the samples. The increased viscosity may signify better binding of the hydrogel samples to the teeth, making it easier to deliver the HAp. The resulting lower $\mathrm{pH}$ caused by the addition of HAp can be an advantage for the hydrogel, as it was shown in previous studies that lower $\mathrm{pH}$ has the tendency to cause higher alteration in dental surfaces due to its effect in remineralization. Dentine tubules were occluded upon treatment with CMC hydrogel containing HAp and layers of crystals were evident in the dentine's surface demonstrating the potential of the fabricated material in treating dental hypersensitivity.

\section{ACKNOWLEDGEMENTS}

This work was supported by the Mid-career Researcher Program through the National Research Foundation of Korea (NRF) grant funded by the Ministry of Education, Science \& Technology (MEST) (NO 20090092808), Republic of Korea and partially supported by Soonchunhyang University Research Fund.

\section{REFERENCES}

[1] Rees, J.S., Jin, L.J., Lam, S., Kudanowska, I. and Vowles, R. (2003) The prevalence of dentine hypersensitivity in a hospital clinic population in Hong Kong. Journal of Den- 
tistry, 31, 453-461. http://dx.doi.org/10.1016/S0300-5712(03)00092-7

[2] Gandolfi, M.G., Iacono, F., Pirani, C. and Prati, C. (2012) The use of calcium-silicate cements to reduce dentine permeability. Archives of Oral Biology, 57, 1054-1061. http://dx.doi.org/10.1016/j.archoralbio.2012.02.024

[3] Gandolfi, M.G., Silvia, F., H, P.D., Gasparotto, G. and Carlo, P. (2008) Calcium silicate coating derived from Portland cement as treatment for hypersensitive dentine. Journal of Dentistry, 36, 565-578. http://dx.doi.org/10.1016/j.jdent.2008.03.012

[4] Wang, Z., Jiang, T., Sauro, S., Pashley, D.H., Toledano, M., Osorio, R., Liang, S., Xing, W., Sa, Y. and Wang, Y. (2011) The dentine remineralization activity of a desensitizing bioactive glass-containing toothpaste: An in vitro study. Australian Dental Journal, 56, 372-381. http://dx.doi.org/10.1111/j.1834-7819.2011.01361.x

[5] Bartold, P.M. (2006) Dentinal hypersensitivity: A review. Australian Dental Journal, 51, 212-218. http://dx.doi.org/10.1111/j.1834-7819.2006.tb00431.x

[6] Clayton, D.R., McCarthy, D. and Gillam, D.G. (2002) A study of the prevalence and distribution of dentine sensitivity in a population of 17 - 58-year-old serving personnel on an RAF base in the Midlands. Journal of Oral Rehabilitation, 29, 14-23. http://dx.doi.org/10.1046/j.1365-2842.2002.00805.x

[7] Curtis, A.R., West, N.X. and Su, B. (2010) Synthesis of nanobioglass and formation of apatite rods to occlude exposed dentine tubules and eliminate hypersensitivity. Acta Biomaterialia, 6, 3740-3746. http://dx.doi.org/10.1016/j.actbio.2010.02.045

[8] Wang, Z., Sa, Y., Sauro, S., Chen, H., Xing, W., Ma, X., Jiang, T. and Wang, Y. (2010) Effect of desensitising toothpastes on dentinal tubule occlusion: A dentine permeability measurement and SEM in vitro study. Journal of Dentistry, 38, 400-410. http://dx.doi.org/10.1016/j.jdent.2010.01.007

[9] Bakry, A.S., Tamura, Y., Otsuki, M., Kasugai, S., Ohya, K. and Tagami, J. (2011) Cytotoxicity of 45S5 bioglass paste used for dentine hypersensitivity treatment. Journal of Dentistry, 39, 599-603.

http://dx.doi.org/10.1016/j.jdent.2011.06.003

[10] Olley, R.C., Pilecki, P., Hughes, N., Jeffery, P., Austin, R. S., Moazzez, R. and Bartlett, D. (2012) An in situ study investigating dentine tubule occlusion of dentifrices following acid challenge. Journal of Dentistry, 40, 585-593. http://dx.doi.org/10.1016/j.jdent.2012.03.008

[11] Miglani, S., Aggarwal, V. and Ahuja, B. (2010) Dentin hypersensitivity: Recent trends in management. Journal of Conservative Dentistry, 13, 218-224. http://dx.doi.org/10.4103/0972-0707.73385

[12] Shen, S.-Y., Tsai, C.-H., Yang, L.-C. and Chang, Y.-C. (2009) Clinical efficacy of toothpaste containing potassium citrate in treating dentin hypersensitivity. Journal of Dental Sciences, 4, 173-177. http://dx.doi.org/10.1016/S1991-7902(09)60023-4

[13] Dababneh, R.H., Khouri, A.T. and Addy, M. (1999) Dentine hypersensitivity: Dentine hypersensitivity [mdash] an enigma? A review of terminology, mechanisms, aetiology and management. British Dental Journal, 187, 606-611. http://dx.doi.org/10.1038/sj.bdj.4800345a

[14] Poggio, C., Lombardini, M., Colombo, M. and Bianchi, S. (2010) Impact of two toothpastes on repairing enamel erosion produced by a soft drink: An AFM in vitro study. Journal of Dentistry, 38, 868-874. http://dx.doi.org/10.1016/j.jdent.2010.07.010

[15] Itthagarun, A., King, N.M. and Cheung, Y.-M. (2010) The effect of nano-hydroxyapatite toothpaste on artificial enamel carious lesion progression: An in-vitro $\mathrm{pH}-\mathrm{cy}-$ cling study. Hong Kong Dental Journal, 7, 6.

[16] Tschoppe, P., Zandim, D. L., Martus, P. and Kielbassa, A. M. (2011) Enamel and dentine remineralization by nanohydroxyapatite toothpastes. Journal of Dentistry, 39, 430437. http://dx.doi.org/10.1016/j.jdent.2011.03.008

[17] Li, L., Pan, H., Tao, J., Xu, X., Mao, C., Gu, X. and Tang, R. (2008) Repair of enamel by using hydroxyapatite nanoparticles as the building blocks. Journal of Materials Chemistry, 18, 4079-4084. http://dx.doi.org/10.1039/b806090h

[18] Kim, Y.S., Kwon, H.K. and Kim, B.I. (2011) Effect of nano-carbonate apatite to prevent re-stain after dental bleaching in vitro. Journal of Dentistry, 39, 636-642. http://dx.doi.org/10.1016/j.jdent.2011.07.002

[19] Domingo, C., Arcís, R. W., Osorio, E., Osorio, R., Fanovich, M.A., Rodríguez-Clemente, R. and Toledano, M. (2003) Hydrolytic stability of experimental hydroxyapatite-filled dental composite materials. Dental Materials, 19, 478-486. http://dx.doi.org/10.1016/S0109-5641(02)00093-3

[20] Liuyun, J., Yubao, L. and Chengdong, X. (2009) Preparation and biological properties of a novel composite scaffold of nano-hydroxyapatite/chitosan/carboxymethyl cellulose for bone tissue engineering. Journal of Biomedical Science, 16, 65. http://dx.doi.org/10.1186/1423-0127-16-65

[21] Chen, H. and Fan, M. (2007) Chitosan/carboxymethyl cellulose polyelectrolyte complex scaffolds for pulp cells regeneration. Journal of Bioactive and Compatible Polymers, 22, 475-491. http://dx.doi.org/10.1177/0883911507081329

[22] Chang, C. and Zhang, L. (2011) Cellulose-based hydrogels: Present status and application prospects. Carbohydrate Polymers, 84, 40-53. http://dx.doi.org/10.1016/j.carbpol.2010.12.023

[23] Miller, J.T., Shannon, I.L., Kilgore, W.G. and Bookman, J.E. (1969) Use of a water-free stannous fluoride-containing gel in the control of dental hypersensitivity. Journal of Periodontology, 40, 490-491. http://dx.doi.org/10.1902/jop.1969.40.8.490

[24] Franco, R., Nguyen, T. and Lee, B.-T. (2011) Preparation and characterization of electrospun PCL/PLGA membranes and chitosan/gelatin hydrogels for skin bioengineering applications. Journal of Materials Science: Materials in Medicine, 22, 2207-2218. http://dx.doi.org/10.1007/s10856-011-4402-8

[25] Heydarzadeh, H.D., Najafpour, G.D. and Nazari-Moghaddam, A. A. (2009) Catalyst-free conversion of alkali cellulose to fine carboxymethyl cellulose at mild conditions. World Applied Sciences Journal, 6, 564-569.

[26] Balamurugan, A., Kannan, S., Selvaraj, V. and Rajeswari, 
S. (2004) Development and spectral characterization of poly(methyl methacrylate)/hydroxyapatite composite for biomedical applications. Trends in Biomaterials and Artificial Organs, 18, 41-45.

[27] Markowitz, K. and Pashley, D.H. (2007) Personal reflections on a sensitive subject. Journal of Dental Research, 86, 292-295. http://dx.doi.org/10.1177/154405910708600401

[28] Meyer-Lueckel, H., Chatzidakis, A.J. and Kielbassa, A.M. (2007) Effect of various calcium/phosphates ratios of carboxymethylcellulose-based saliva substitutes on mineral loss of bovine enamel in vitro. Journal of Dentistry, 35, 851-857. http://dx.doi.org/10.1016/j.jdent.2007.08.006

[29] Tongdeesoontorn, W., Mauer, L., Wongruong, S., Sriburi, P. and Rachtanapun, P. (2011) Effect of carboxymethyl cellulose concentration on physical properties of biodegradable cassava starch-based films. Chemistry Central Journal, 5, 6. http://dx.doi.org/10.1186/1752-153X-5-6

[30] Simonzadeh, N. and Ronsen, B. (2012) An isocratic HPLC method for the determination of sorbitol and glycerol in pharmaceutical formulations. Journal of Chromatographic Science, 50, 644-647. http://dx.doi.org/10.1093/chromsci/bms044

[31] Padilla, S., García-Carrodeguas, R. and Vallet-Regí, M. (2004) Hydroxyapatite suspensions as precursors of pieces obtained by gelcasting method. Journal of the European Ceramic Society, 24, 2223-2232. http://dx.doi.org/10.1016/S0955-2219(03)00629-0

[32] Hilgenberg, S.P., Pinto, S.C.S., Farago, P.V., Santos, F.A. and Wambier, D.S. (2011) Physical-chemical characteristics of whitening toothpaste and evaluation of its effects on enamel roughness. Brazilian Oral Research, 25, 288-
294. http://dx.doi.org/10.1590/S1806-83242011005000012

[33] Thomann, J.M., Voegel, J.C. and Gramain, P. (1990) Kinetics of dissolution of calcium hydroxyapatite powder. III: $\mathrm{pH}$ and sample conditioning effects. Calcified Tissue International, 46, 121-129. http://dx.doi.org/10.1007/BF02556096

[34] Chung, M.K., Jue, S.S. and Dong, X. (2012) Projection of non-peptidergic afferents to mouse tooth pulp. Journal of Dental Research, 91, 777-782. http://dx.doi.org/10.1177/0022034512450298

[35] Boonanantanasarn, K., Janebodin, K., Suppakpatana, P., Arayapisit, T., Rodsutthi, J.-A., Chunhabundit, P., Boonanuntanasarn, S. and Sripairojthikoon, W. (2012) $<\mathrm{i}>$ Morinda citrifolia $</ \mathbf{i}>$ leaves enhance osteogenic differentiation and mineralization of human periodontal ligament cells. Dental Materials Journal, 31, 863-871.

[36] Imazato, S., Horikawa, D., Ogata, K., Kinomoto, Y. and Ebisu, S. (2006) Responses of MC3T3-E1 cells to three dental resin-based restorative materials. Journal of Biomedical Materials Research Part A, 76, 765-772. http://dx.doi.org/10.1002/jbm.a.30422

[37] Featherstone, J.D.B. (2000) The science and practice of caries prevention. The Journal of the American Dental Association, 131, 887-899.

[38] Tilliss, T.S. and Keating, J.G. (2002) Understanding and managing dentin hypersensitivity. Journal of Dental Hygiene, 76, 296-310.

[39] Rösing, C.K., Fiorini, T., Liberman, D.N. and Cavagni, J. (2009) Dentine hypersensitivity: Analysis of self-care products. Brazilian Oral Research, 23, 56-63. http://dx.doi.org/10.1590/S1806-83242009000500009 